\title{
Modified FLOX as first-line chemotherapy for metastatic colorectal cancer patients in the public health system in Brazil: Effectiveness and cost-utility analysis
}

\author{
DANIELA R. NEBULONI ${ }^{1,2}$, MILENA P. MAK ${ }^{1,2}$, FABIANO HAHN SOUZA ${ }^{1,2}$, DANIEL F. SARAGIOTTO ${ }^{1,3}$, \\ THIAGO JÚLIO ${ }^{1-3}$, GILBERTO DE CASTRO Jr ${ }^{1,2}$, JORGE SABBAGA ${ }^{1}$ and PAULO M. HOFF $^{1-3}$ \\ ${ }^{1}$ Clinical Oncology Department, Cancer Institute of the State of Sao Paulo (ICESP), CEP 01246-000; \\ ${ }^{2}$ Clinical Oncology Department, University of Sao Paulo Medical School, CEP 01246-903; \\ ${ }^{3}$ Oncology Center, Sírio-Libanês Hospital, CEP 01308-050, Sao Paulo, SP, Brazil
}

Received May 18, 2012; Accepted July 24, 2012

DOI: $10.3892 / \mathrm{mco} .2012 .12$

\begin{abstract}
Incorporation of new drugs for treatment of metastatic colorectal cancer (mCRC) has led to a clear improvement in overall patient survival, the added cost of treatment, however, is a major concern worldwide. The cost-effectiveness of using a modified FLOX (mFLOX) regimen for treating $\mathrm{mCRC}$ patients was delineated. In this study, 82 consecutive mCRC patients were treated with leucovorin (LV) at $20 \mathrm{mg} / \mathrm{m}^{2}$ in combination with weekly bolus of 5-fluorouracil (5-FU) $\left(500 \mathrm{mg} / \mathrm{m}^{2}\right)$ for 6 consecutive weeks and oxaliplatin $\left(85 \mathrm{mg} / \mathrm{m}^{2}\right)$ at weeks 1,3 and 5 , every 8 weeks. Overall survival (OS) and toxicity were evaluated. A Markov Model with a 2-year time horizon and 2-week cycles was developed, comparing mFLOX and mFOLFOX6 in a Brazilian environment. Health outcomes were measured in quality-ajusted life years (QALYs). The median overall period of survival was 19 months, while the estimated 1-year survival was $75 \%$. Response by RECIST was assessed in 33 patients. Partial response was observed in $39.4 \%$ of patients, while $36.3 \%$ were stable. The mFLOX regimen cost was BRL 9,000, while the mFOLFOX6 BRL 22,000 (1 EUR=2.29 BRL), leading to an incremental costed of BRL 13,000, considering a 20-week period of first-line therapy. The incremental effect of the mFOLFOX was of 0,117 QALY. The incremental costeffectiveness ratio of mFOLFOX6 was of BRL 110,344/QALY. The sensitivity analysis detected no differences in the outcome measures. In conclusion, the mFLOX is an active regimen in
\end{abstract}

Correspondence to: Dr Milena P. Mak, Clinical Oncology Department, Cancer Institute of the State of Sao Paulo (ICESP), Avenida Dr. Arnaldo 251, 5th Floor, CEP 01246-000, Sao Paulo, SP, Brazil

E-mail: milena.mak@icesp.org.br

Key words: colorectal cancer, cost-utility, oxaliplatin, leucovorin, 5-fluorouracil
mCRC patients, possibly providing a cost-effective option in public health systems.

\section{Introduction}

Colorectal cancer (CRC) is the second most prevalent cancer in the world. In Brazil, approximately 30,000 new CRC cases occur annually, while according to national data, $24 \%$ of the CRC cases are metastatic at the time of diagnosis (1).

5-Fluorouracil (5-FU) was the first medication approved for treating patients with metastatic colorectal cancer (mCRC) and remains the medication of choice for single-agent chemotherapy. However, for optimal 5-FU efficacy, a combination with leucovorin (LV) is required. LV increases the intracellular concentration of methylene tetrahydrofolate, thus stabilizing the ternary complex formed between the fluorodeoxyuridine monophosphate (FdUMP) and the thymidylate synthase (TS), improving the cytotoxic activity of 5-FU (2). Modulation of 5-FU by LV significantly improves the response rate compared to 5-FU alone. Several doses of LV have already been tested in clinical trials and, compared to a low dose $\left(20 \mathrm{mg} / \mathrm{m}^{2}\right)$, higher doses (200-500 mg/m ${ }^{2}$ ) of LV are more expensive and toxic, while producing comparable figures in response rate (RR), progression-free survival (PFS) and overall survival (OS) (2).

Attempts to improve survival and response rates have led to the incorporation of new drugs in the chemotherapeutic armamentarium for mCRC patients. A gradual increase in PFS and OS has been achieved by introducing cytotoxic agents, such as oxaliplatin and irinotecan, either in first- or second-line therapy $(3,4)$. Even better results have been achieved by combining cytotoxic medications with biological agents. Bevacizumab and cetuximab are monoclonal antibodies directed at the vascular endothelial growth factor (VEGF) and epidermal growth factor receptor (EGFR), respectively. Various associations of 5-FU with one or more of these new agents are now considered valid therapeutic options for mCRC patients (5-7).

However, a significant increment in chemotherapy costs has been a major concern. The introduction of new regimens for treating mCRC has led to a 340-fold increase in the cost of therapy compared to traditional 5-FU-based treatment 
(8). Several protocols based on oxaliplatin associated with capecitabine or with continuously infused 5-FU have already been thoroughly analyzed, including FOLFOX4, FOLFOX6, FUFOX, OXAFAFU, XELOX, CAPOX and OXXE, leading to similar efficacy results in first-line therapy, justifying numerous discussions regarding their cost-effectiveness (9). However, the bolus administration of 5-FU in the treatment of mCRC patients is a reality in developing countries, including Brazil, where $90 \%$ of the population utilizes the public health system (10). Portable infusion pumps, implantable catheters or the substitution of 5-FU with capecitabine would indeed add prohibitively to treatment costs.

Based on previously reported data, the Instituto do Cancer do Estado de Sao Paulo (ICESP) (Sao Paulo, Brazil), a recognized public oncology center in Brazil, adopted a modified FLOX (mFLOX) protocol for first-line treatment of mCRC in May 2007. FLOX, a regimen described for adjuvant intention, traditionally requires weekly administration of $500 \mathrm{mg}$ / $\mathrm{m}^{2}$ of LV (11). mFLOX is essentially the same as FLOX with regard to the schedule and doses of oxaliplatin and 5-FU. However, a much lower dose $\left(20 \mathrm{mg} / \mathrm{m}^{2}\right)$ of $\mathrm{LV}$ was administered in this regimen, reducing the cost associated with treatment. The present study analyzed the results of using mFLOX in our facility, as well as the cost-effectiveness of this regimen.

\section{Materials and methods}

Study design and population. A retrospective analysis was carried out, using electronic charts of 82 consecutive patients treated with mFLOX as a first-line therapy for mCRC, between May, 2007 and October, 2009. Our primary goal was to determine patients' overall survival. Response rate and toxicity were also assessed as a secondary analysis. In conjunction, an exploratory analysis of mFLOX cost-effectiveness was conducted.

Dose administration. This regimen included the traditional FLOX protocol described in the NSABP C-07 trial, except for the reduction of the $\mathrm{LV}$ dose to $20 \mathrm{mg} / \mathrm{m}^{2}$ (11). Therefore, a weekly bolus of 5 -FU $\left(500 \mathrm{mg} / \mathrm{m}^{2}\right)$ in combination with LV $\left(20 \mathrm{mg} / \mathrm{m}^{2}\right)$ was administered for 6 consecutive weeks, and oxaliplatin $\left(85 \mathrm{mg} / \mathrm{m}^{2}\right)$ was administered in weeks 1,3 and 5 , every 8 weeks. Emesis prophylaxis with dexamethasone $(20 \mathrm{mg})$ and ondansetron $(8 \mathrm{mg})$ was administered prior to oxaliplatin infusion. Patients were treated until progressive disease, unacceptable toxicity or absence of additional benefit were diagnosed. 5-FU and oxaliplatin doses were reduced by $20 \%$ in grade 3 or 4 toxicities.

Response assessment. Evaluation scans were retrospectively blind-reviewed by two experienced radiologists, using RECIST version 1.1 (12).

Statistical analysis. OS was defined as the time between metastatic cancer diagnosis and death by any cause and was estimated by the Kaplan-Meier method. The analyses were carried out on an intent to treat basis. Toxicities were graded according to the National Cancer Institute criteria, version 3.0 (13).
Table I. Utilities.

\begin{tabular}{lc}
\hline Health state & QALY \\
\hline Stable disease (first-line CT) & 0.50 \\
Progression of disease & 0.29 \\
Best supportive care & 0.21 \\
Neutropenic fever G3/4 & 0.46 \\
Peripheral neuropathy G3/4 & 0.40 \\
Diarrhea G3/4 & 0.32 \\
Mortality & 0
\end{tabular}

QALY, quality-adjusted life year; G3/4, grade 3/4; CT, chemotherapy.

Table II. Resource utilization by Markov Model (two-week period).

BRL EUR

\section{Chemotherapy}

$\begin{array}{lrr}\text { FLOX }^{\mathrm{a}} & 2,587 & 1,130 \\ \text { mFOLFOX }^{\mathrm{a}} & 5,161 & 2,254 \\ \text { Irinotecan monotherapy }^{\mathrm{a}} & 1,274 & 556 \\ \text { Hospitalization (rate/day) } & & \\ \text { Inpatient ward } & 1,098 & 479 \\ \text { Intensive care unit } & 3,252 & 1,420 \\ \text { Emergency service } & 694 & 303 \\ \text { Grade 3/4 toxicities } & & \\ \text { Diarrhea } & 3,613 & 1,578 \\ \text { Neuropathy } & 869 & 379 \\ \text { Neutropenic fever } & 9,395 & 4,103\end{array}$

${ }^{\mathrm{a}}$ Eight-week treatment.

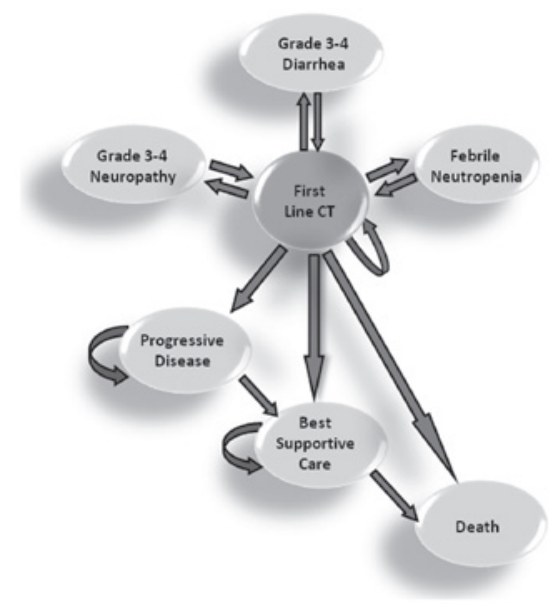

Figure 1. Markov Model. CT, chemotherapy.

Evaluation of cost-effectiveness using the Markov Model. To evaluate the cost-effectiveness of mFLOX in comparison to mFOLFOX6, a Markov Model with a 2-year time horizon 
and 2-week cycles was developed (Fig. 1). Probabilities of toxicities (neutropenia, diarrhea and neuropathy), progressive disease, overall survival and likelihood of second-line therapy were based on published literature and data obtained from the retrospective analysis carried out. It was evaluated that $58 \%$ (14) of patients assigned to mFOLFOX6 and $44.6 \%$ of patients on mFLOX would be eligible for a second-line therapy based on irinotecan monotherapy $\left(350 \mathrm{mg} / \mathrm{m}^{2}\right.$ intravenously on day 1 every 3 weeks). Utilities were based on the available literature data (Table I). Costs of the physician, hospital services and medication were obtained from DATASUS, ANVISA and local resources (Table II). The analysis adhered to the Brazilian government policies. Health outcomes were measured in quality-adjusted life years (QALYs). To address uncertainty in model parameters, one-way sensibility analyses were carried out. Costs and utilities were discounted at $5 \%$. mFLOX was assumed to be $20 \%$ inferior to mFOLFOX6 in terms of efficacy, while the median time of first-line therapy was 20 weeks.

This study was approved by the Institutional Review Board and was conducted in compliance with Brazilian law.

\section{Results}

Patients and tumor characteristics. As previously described, 82 patients were retrospectively evaluated. The mean age of the patients was 54 years (range, 41-70), with $41(50 \%)$ patients being male. In $51(62.2 \%)$ patients the primary tumor site was the colon, while in 31 (37.8\%) the rectum. Sixty patients $(73.2 \%)$ had received adjuvant or neo-adjuvant treatment. Eighteen patients $(11 \%)$ had no previous resection of the primary tumor. Thirty-three patients (40.2\%) were submitted to surgery with curative, and 31 (48.4\%) with palliative intent. The most frequent metastatic sites were the liver (73.2\%), the lungs (29.3\%), the peritoneum (29.3\%) and the lymph nodes $(26.8 \%)$. The mean number of metastatic sites was 2 . Twenty-eight (34\%) patients had 1, $38(46 \%)$ had 2, while 3 (19\%) patients had 3 metastatic sites. The majority of patients demonstrated a good performance status, $66(80 \%)$ patients were classified as ECOG 0-1, while $8(10 \%)$ patients as ECOG 2-3. The median treatment time was 138 days (range, 0-409) and the median number of cycles administered was 2.5 (range, 0-6). These data are summarized in Table III.

Toxicity profile. Eighty-one patients were analyzed for toxicity (Table IV). Diarrhea occurred in 39 (48\%) patients and was graded as 3 or 4 in 17 (21\%) patients. One death occurred due to grade 5 diarrhea. Neurological toxicity, represented by peripheral neuropathy, occurred in 28 (34.6\%) patients, although grade 3 was observed only in $2(2.5 \%)$ patients. Grade 3 hematological toxicity occurred in 4 (4.9\%), while grade 4 in $5(6.2 \%)$ patients. Mucositis was an infrequent complication, occurring only in $4(4.9 \%)$ patients. Twenty-five (30\%) patients needed chemotherapy dose reduction.

Response to treatment and survival. Response by RECIST was assessed in 33 patients, due to non-homogeneously performed CT scans resulting in some patients not having a baseline CT scan in our institution. Partial response (PR) was observed in
Table III. Baseline characteristics of the patients $(n=82)$.

\begin{tabular}{|c|c|}
\hline Patients & No. $(\%)$ \\
\hline \multicolumn{2}{|l|}{ Age (years) } \\
\hline Mean & 54 \\
\hline Range & $41-70$ \\
\hline \multicolumn{2}{|l|}{ Gender } \\
\hline Male & $41(50.0)$ \\
\hline Female & $41(50.0)$ \\
\hline \multicolumn{2}{|l|}{ Primary site } \\
\hline Colon & $51(62.2)$ \\
\hline Rectum & $31(37.8)$ \\
\hline \multicolumn{2}{|l|}{$\mathrm{T}$ staging } \\
\hline $\mathrm{T} 2$ & $6(7.3)$ \\
\hline $\mathrm{T} 3$ & $49(59.8)$ \\
\hline $\mathrm{T} 4$ & $13(15.9)$ \\
\hline NA & $14(17.0)$ \\
\hline \multicolumn{2}{|l|}{$\mathrm{N}$ staging } \\
\hline NO & $21(25.6)$ \\
\hline N1 & $21(25.6)$ \\
\hline $\mathrm{N} 2$ & $20(24.4)$ \\
\hline N3 & $2(2.4)$ \\
\hline NA & $18(22.0)$ \\
\hline \multicolumn{2}{|l|}{ ECOG performance status } \\
\hline 0 & $24(29.2)$ \\
\hline 1 & $42(51.2)$ \\
\hline 2 & $5(6.1)$ \\
\hline 3 & $3(3.7)$ \\
\hline NA & $8(9.8)$ \\
\hline \multicolumn{2}{|l|}{ Site of metastasis } \\
\hline Liver & $60(73.2)$ \\
\hline Lung & $24(29.3)$ \\
\hline Peritoneum & $24(29.3)$ \\
\hline Lymph nodes & $22(26.8)$ \\
\hline Bone & $2(2.4)$ \\
\hline Ovary & $5(6.1)$ \\
\hline Central nervous system & $1(1.2)$ \\
\hline Other & $1(17.1)$ \\
\hline \multicolumn{2}{|l|}{ No. of metastatic sites } \\
\hline 1 & $28(34.1)$ \\
\hline 2 & $38(46.3)$ \\
\hline 3 & $16(19.5)$ \\
\hline \multicolumn{2}{|l|}{ Previous treatment } \\
\hline Yes & $60(73.2)$ \\
\hline No & $22(26.8)$ \\
\hline \multicolumn{2}{|l|}{ Type of previous treatment } \\
\hline Chemotherapy & $12(14.6)$ \\
\hline Chemo-radiotherapy & $16(19.5)$ \\
\hline Median number of mFLOX cycles & 2.5 \\
\hline Range & $(0-6)$ \\
\hline Median days in treatment & 138 \\
\hline Range & $(0-409)$ \\
\hline \multicolumn{2}{|l|}{ Resection to primary site } \\
\hline Curative & $33(40.2)$ \\
\hline Palliative & $31(48.4)$ \\
\hline
\end{tabular}


Table IV. Toxicities secondary to mFLOX treatment.

\begin{tabular}{lcccccc}
\hline & \multicolumn{5}{c}{ Grade, $\mathrm{n}(\%)$} \\
\cline { 2 - 6 } Toxicity & 0 & 1 & 2 & 3 & 4 & 5 \\
\hline Diarrhea & $42(51.9)$ & $13(16.0)$ & $8(9.9)$ & $5(6.2)$ & $12(14.8)$ & $1(1.2)$ \\
Mucositis & $77(95.1)$ & $2(2.5)$ & - & $1(1.2)$ & $1(1.2)$ & - \\
Neurological & $53(65.4)$ & $20(24.7)$ & $6(7.4)$ & $2(2.5)$ & - & - \\
Hematological & $55(67.9)$ & $6(7.4)$ & $11(13.6)$ & $4(4.9)$ & $5(6.2)$ & - \\
\hline
\end{tabular}

Patients, $\mathrm{n}=81$. Toxicity information was lacking for 1 patient.

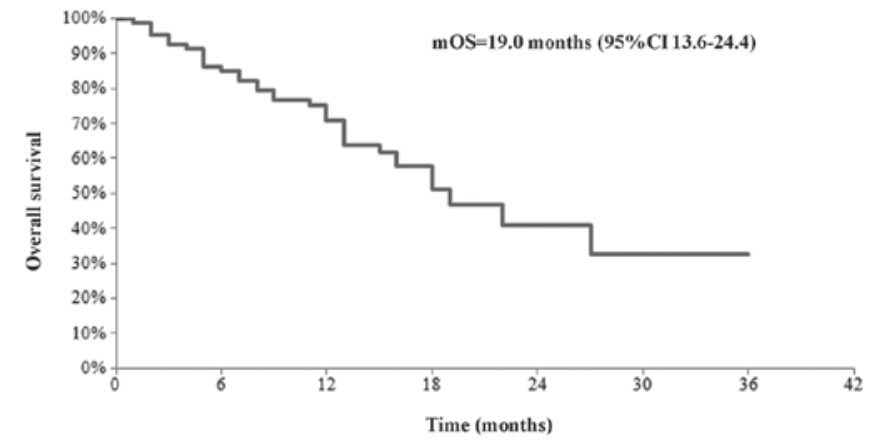

Figure 2. Overall survival of mCRC patients submitted to mFLOX. mCRC, metastatic colorectal cancer; mFLOX, modified FLOX.

Table V. Comparison of the dose of chemotherapeutic agents in $\mathrm{mFLOX}$, bFOL and N-FLOX.

\begin{tabular}{lrrr}
\hline Chemotherapy $^{\mathrm{a}}$ & mFLOX & bFOL & N-FLOX \\
\hline Oxaliplatin & 255 & 340 & 340 \\
5-FU & 3000 & 3000 & 4000 \\
LV & 120 & 120 & 480 \\
Dose/week & & & \\
Oxaliplatin & 31.9 & 42.5 & 42.5 \\
5-FU & 375.0 & 375.0 & 500 \\
LV & 15 & 15 & 60 \\
\hline
\end{tabular}

${ }^{\mathrm{a} D o s e}$ total in 8 weeks $\left(\mathrm{mg} / \mathrm{m}^{2}\right)$. mFLOX, modified FLOX; 5-FU, 5-fluorouracil and LV, leucovorin.

$39.4 \%$ of these patients, with $36.3 \%$ being stable. Progression disease (PD) occurred in 8 (24.2\%) patients.

Patients were analyzed in terms of survival. The median OS was 19.0 months (95\% CI, 13.6-24.4 months) (Fig. 4). The estimated 1-year survival was $\sim 75 \%$.

Second-line treatment. Eighteen patients (22\%) were lost to follow-up. Sixty-four patients were analyzed for second-line treatment. Thirty-six (44\%) patients received second-line irinotecan-based chemotherapy (irinotecan monotherapy $350 \mathrm{mg} / \mathrm{m}^{2}$, every 21 days) on PD, while 3 patients $(4 \%)$ received mitomycin-based chemotherapy as third-line treat-

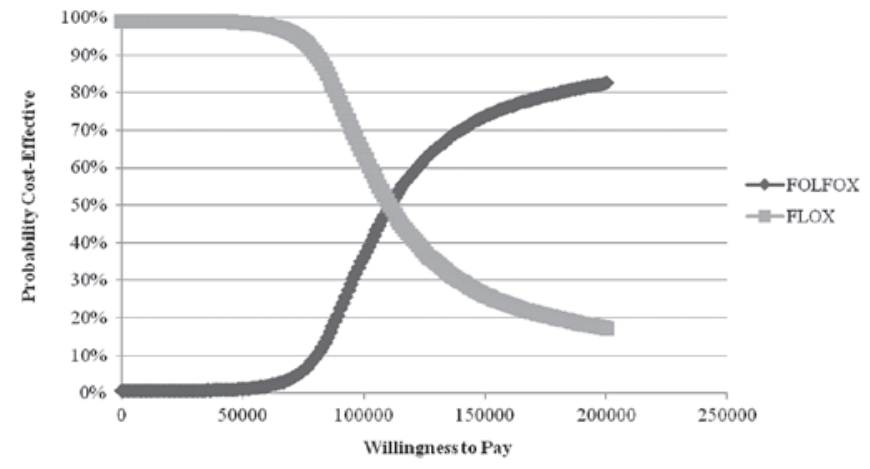

Figure 3. Acceptability curve (10,000 probabilistic Monte Carlo simulations).

ment. Subsequent to a period with no chemotherapy or with 5-FU/LV alone, 3 patients (4\%) received mFLOX again upon PD. Eight patients $(10 \%)$ received chemotherapy with only $5-\mathrm{FU} / \mathrm{LV}$, while 10 patients $(12 \%)$ received best supportive care only. Three patients $(4 \%)$ underwent resection for metastatic disease.

Cost-effectiveness analysis. The mFLOX regimen had a cost of BRL 9,000 (1 EUR=2.29 BRL) and mFOLFOX6 BRL 22,000 leading to an incremental cost of BRL 13,000, considering a 20 -week duration of first-line therapy in a 14-day cycle. The incremental effect of mFOLFOX6 was of 0.117 QALY. The costeffectiveness of mFLOX was BRL 69,889, with mFOLFOX6 being BRL 88,654. The incremental cost-effectiveness ratio of mFOLFOX6 was BRL 110,344/QALY. In 10,000 probabilistic Monte Carlo simulations, mFLOX was cost effective in $60 \%$ of trials using a threshold of BRL 100,000/QALY (Fig. 3). The sensitivity analysis detected no differences in outcome measures.

\section{Discussion}

With a view to minimize costs for treating mCRC patients, a new chemotherapy regimen avoiding central lines and infusion pumps and utilizing small doses of LV was routinely used in the present study. Following this mFLOX protocol, a median OS of 19 months was achieved outside of a clinical trial. This figure is comparable to those obtained with more expensive oxaliplatin-containing regimens, such as mFOLFOX6 producing a median OS of 20.2 months and a median PFS of 
8.9 months (4). In the present cost-effectiveness analysis, a 5-FU infusion regimen as mFOLFOX6 had an increment in cost-effectiveness of BRL 110,344/QALY, which is above the World Health Organization threshold of three times the Gross Domestic Product per Capita (BRL 53,871 for Brazil) (15).

Other bolus regimens based on oxaliplatin, with no associated biologic agent, have already been described. The bFOL protocol consisting of oxaliplatin $\left(85 \mathrm{mg} / \mathrm{m}^{2}\right)$ every other week coupled with $\mathrm{LV}\left(20 \mathrm{mg} / \mathrm{m}^{2}\right)$ and 5 -FU $\left(500 \mathrm{mg} / \mathrm{m}^{2}\right)$ every week, for three consecutive weeks in a four-week cycle, was tested on 42 patients with untreated mCRC and the results were similar: the OS was 15.9 months (95\% CI, 11.4-19.7 months), and the median time to progression (TTP) was 9.0 months (95\% CI, 7.1-10.8 months). Complete response and PR were observed in 3 and 23 patients, respectively (16). The Nordic-FLOX (N-FLOX) regimen was tested as a first-line treatment in 85 patients with mCRC. The scheme consisted of bi-weekly cycles of oxaliplatin $\left(85 \mathrm{mg} / \mathrm{m}^{2}\right)$ administered on Day 1 , followed by bolus infusion of 5-FU $\left(500 \mathrm{mg} / \mathrm{m}^{2}\right)$ and $\mathrm{LV}\left(60 \mathrm{mg} / \mathrm{m}^{2}\right) .5-\mathrm{FU}$ coupled with LV alone was repeated on Day 2. The median OS was 16.1 months (95\% CI, 12.7-19.6 months), and the median TTP was 7.0 months (95\% CI, 6.3-7.7 months) (17). Notably, the dose-intensity of oxaliplatin, 5-FU and LV differs in each of these regimens, possibly accounting for the differences in the response rate and toxicity. Without delay between cycles, the total dose received by patients undergoing chemotherapy in each 8-week treatment-cycle of mFLOX, bFOL and N-FLOX varies considerably (Table V). The higher dose of oxaliplatin seems more relevant in terms of response rate compared to the higher doses of 5-FU and LV, which are probably more correlated with the toxicity profile.

The present study was of retrospective nature, impairing accuracy in determining the response rate obtained using mFLOX. However, survival data are abundant, in an environment of patients treated in a large public cancer hospital. Peritoneum metastasis was present in 24 patients $(29 \%)$. Sixteen patients $(19.5 \%)$ had three different sites of metastasis, while one patient had secondary lesions in the central nervous system.

In conclusion, the findings showed that mFLOX is an active, cost-effective and convenient regimen for treating mCRC, possibly providing another low-cost option for public health systems in developing countries.

\section{Acknowledgements}

The authors are indebted to Alberto Sobrero for his valuable comments and to Julia Nakashima for her statistical assistance in analyzing the data.

\section{References}

1. Instituto Nacional de Câncer: Estimativa 2010, Incidência de Câncer no Brasil. http://www1.inca.gov.br/estimativa/2010. Accessed November, 2011

2. Jager E, Heike M, Bernhard H, et al: Weekly high-dose leucovorin versus low-dose leucovorin combined with fluorouracil in advanced colorectal cancer: results of a randomized multicenter trial. Study Group for Palliative Treatment of Metastatic Colorectal Cancer Study Protocol 1. J Clin Oncol 14: 2274-2279, 1996.
3. Tournigand C, Andre T, Achille E, et al: FOLFIRI followed by FOLFOX6 or the reverse sequence in advanced colorectal cancer: a randomized GERCOR study. J Clin Oncol 22: 229-237, 2004.

4. Sanoff HK, Sargent DJ, Campbell ME, et al: Five-year data and prognostic factor analysis of oxaliplatin and irinotecan combinations for advanced colorectal cancer: N9741. J Clin Oncol 26: 5721-5727, 2008 .

5. Hurwitz H, Fehrenbacher L, Novotny W, et al: Bevacizumab plus irinotecan, fluorouracil, and leucovorin for metastatic colorectal cancer. N Engl J Med 350: 2335-2342, 2004.

6. Van Cutsem E, Kohne CH, Hitre E, et al: Cetuximab and chemotherapy as initial treatment for metastatic colorectal cancer. N Engl J Med 360: 1408-1417, 2009.

7. Oliveira SC, Machado KK, Sabbaga J and Hoff PM: Integration of anti-vascular endothelial growth factor therapies with cytotoxic chemotherapy in the treatment of colorectal cancer. Cancer J 16: 220-225, 2010.

8. Ferro SA, Myer BS, Wolff DA, et al: Variation in the cost of medications for the treatment of colorectal cancer. Am J Manag Care 14: 717-725, 2008.

9. Douillard JY, Bennouna J and Senellart H: Is XELOX equivalent to FOLFOX or other continuous-infusion 5-fluorouracil chemotherapy in metastatic colorectal cancer? Clin Colorectal Cancer 7: 206-211, 2008.

10. Ministério da Saúde: SUS - 15 anos de implantação: desafios e propostas para a sua consolidação. Ministério da Saúde, Brasil, 2003.

11. Kuebler JP, Wieand HS, O'Connell MJ, et al: Oxaliplatin combined with weekly bolus fluorouracil and leucovorin as surgical adjuvant chemotherapy for stage II and III colon cancer: results from NSABP C-07. J Clin Oncol 25: 2198-2204, 2007.

12. Eisenhauer EA, Therasse P, Bogaerts J, et al: New response evaluation criteria in solid tumours: revised RECIST guideline (version 1.1). Eur J Cancer 45: 228-247, 2009.

13. National Cancer Institute: National Cancer Institute Common Toxicity Criteria. http://ctep.cancer.gov/reporting/ctc.html. Accessed November, 2011

14. Colucci G, Gebbia V, Paoletti G, et al: Phase III randomized trial of FOLFIRI versus FOLFOX4 in the treatment of advanced colorectal cancer: a multicenter study of the Gruppo Oncologico Dell'Italia Meridionale. J Clin Oncol 23: 4866-4875, 2005.

15. World Health Organization (WHO) CHOosing Interventions that are Cost Effective (WHO-CHOICE): Threshold values for intervention cost-effectiveness by region (tables listing prices and other relevant information for cost analysis). 2011.

16. Hochster H, Chachoua A, Speyer J, Escalon J, ZeleniuchJacquotte A and Muggia F: Oxaliplatin with weekly bolus fluorouracil and low-dose leucovorin as first-line therapy for patients with colorectal cancer. J Clin Oncol 21: 2703-2707, 2003.

17. Sorbye H, Glimelius B, Berglund A, et al: Multicenter phase II study of Nordic fluorouracil and folinic acid bolus schedule combined with oxaliplatin as first-line treatment of metastatic colorectal cancer. J Clin Oncol 22: 31-38, 2004. 